\title{
Multifunctional hypoxia-involved gene silencing nanoplatform for sensitizing photochemotherapy
}

Huimin Wang, ${ }^{\dagger 1}$ Yuqi Chen, ${ }^{\dagger 2}$ Jinhua Shang, ${ }^{1}$ Hong Wang, ${ }^{1}$ Min Pan,${ }^{1}$ Xiaoqing Liu, ${ }^{1}$ Xiang Zhou, ${ }^{2}$ Fuan Wang*1

${ }^{1}$ Key Laboratory of Analytical Chemistry for Biology and Medicine (Ministry of Education), College of Chemistry and Molecular Sciences, Wuhan University, Wuhan 430072, P. R. China.

${ }^{2}$ Key Laboratory of Biomedical Polymers (Ministry of Education), College of Chemistry and Molecular Sciences, Wuhan University, 430072 Wuhan, P. R. China.

* To whom correspondence should be addressed. E-mail: fuanwang@,whu.edu.cn

$\uparrow$ These authors contributed equally to this work 


\section{Table of Contents}

Figure S1. SEM characterization of different nanoparticles........................................ S-2

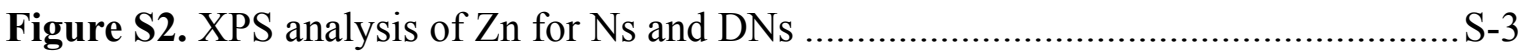

Figure S3. The size analysis of CDHNs in different solution.................................... S-4

Figure S4. UV-vis absorbance spectra of Ce6 and DOX in the presence of zinc ions ..... S-5

Figure S5. The characteristic UV-vis absorption peak of siRNA ….............................. S-6

Figure S6. The drug loading capacity of different payloads....................................... S-8

Figure S7. The effect of ROS on the integrity of siRNA in CDHNs.............................. S-9

Figure S8. Cytotoxicity of CNs on MCF-10A and MDR/MCF-7 cells...................... S-10

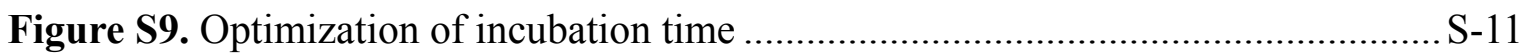

Figure S10. Intracellular uptake of CNs by CLSM ................................................. 12

Figure S11. The accelerated lysosome escape of Ce6 under irradiation .................... S-13

Figure S12. The accelerated release of DOX by PDT ..............................................

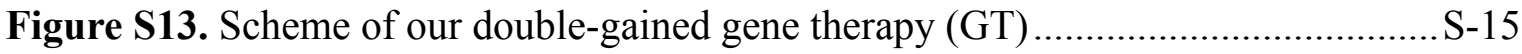

Figure S14. The annexin V-FITC/PI-staining method .......................................... S-16

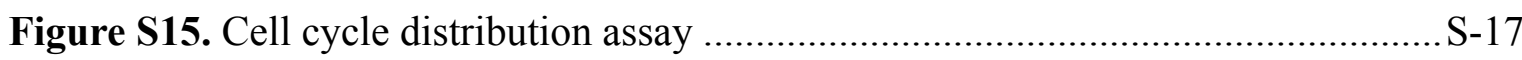

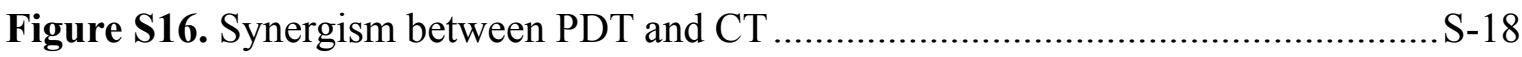

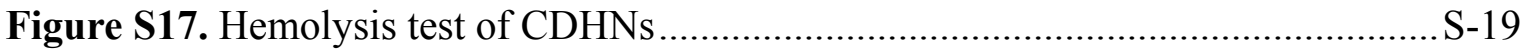

Figure S18. H\&E staining of CDHNs in major organs .......................................... S-20

Figure S19. Serum biochemistry analysis of CDHNs ........................................... S-2 1

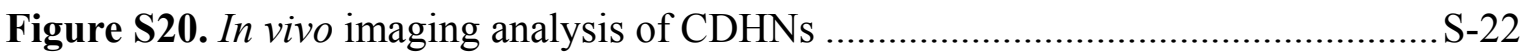

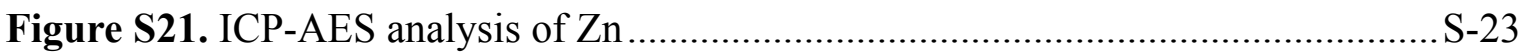

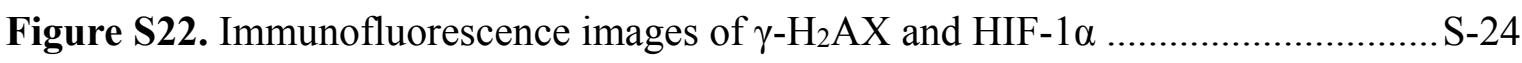




\section{SEM characterization of different nanoparticles}

Different agents were encapsulated into ZIF-8 through the all in one process. The scanning electron microscopy (SEM) images of different nanoparticles showed uniform

spherical-like morphology as that of pure ZIF-8 nanoparticles, indicating that the encapsulation of different payloads had no effect on their morphological features.

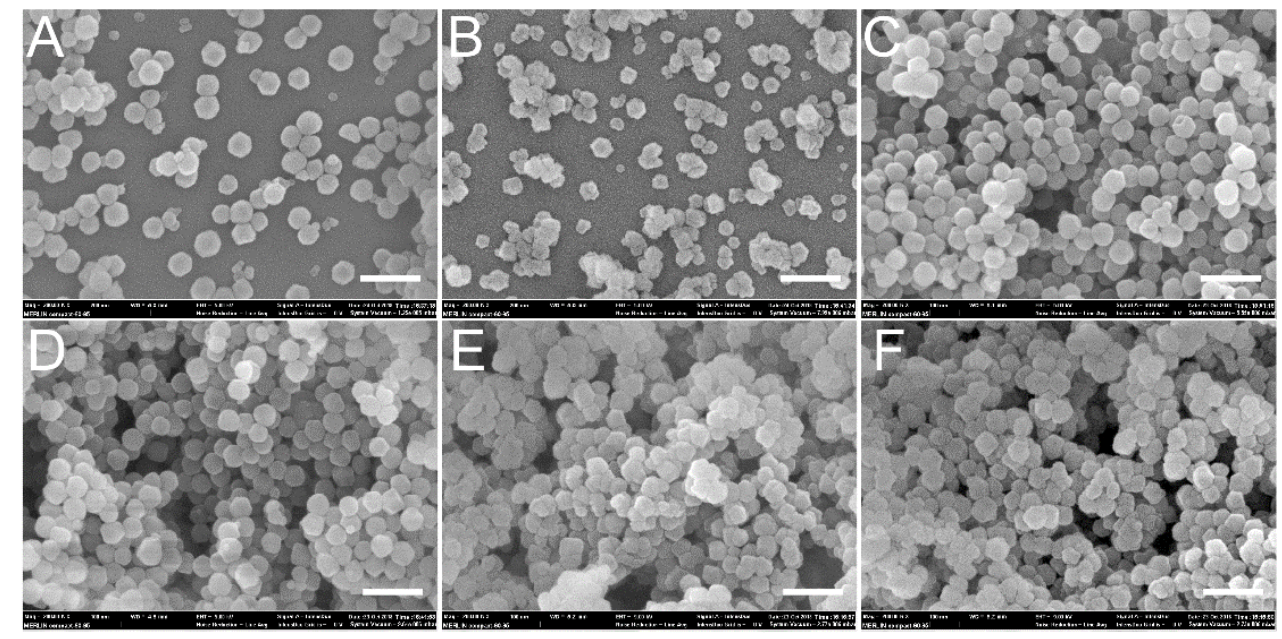

Figure S1. SEM characterization of different nanoparticles: (A) Ns, (B) DNs, (C) CNs, (D) CHNs, (E) CDNs, (F) CDHNs. Scale bar $=200 \mathrm{~nm}$. 


\section{XPS analysis of Zn for ZIF-8 and DNs}

The coordination interactions between $\mathrm{Zn}^{2+}$ and DOX were investigated by using X-ray photoelectron spectroscopy (XPS). As compared with ZIF-8 NPs (Ns), the characteristic binding energy peaks of $\mathrm{Zn} 2 \mathrm{p}_{1 / 2}$ and $\mathrm{Zn} 2 \mathrm{p}_{3 / 2}$ were respectively shifted + $0.2 \mathrm{eV}$ (from $1044.3 \mathrm{eV}$ to $1044.5 \mathrm{eV}$ ) and $+0.1 \mathrm{eV}$ (from $1021.3 \mathrm{eV}$ to $1021.4 \mathrm{eV}$ ) for DOX-encapsulated ZIF-8 NPs (DNs), Figure S2. This varied binding energy might be attributed to the electron transfer from the encapsulated DOX to the active $\mathrm{Zn}$ sites of ZIF-8.

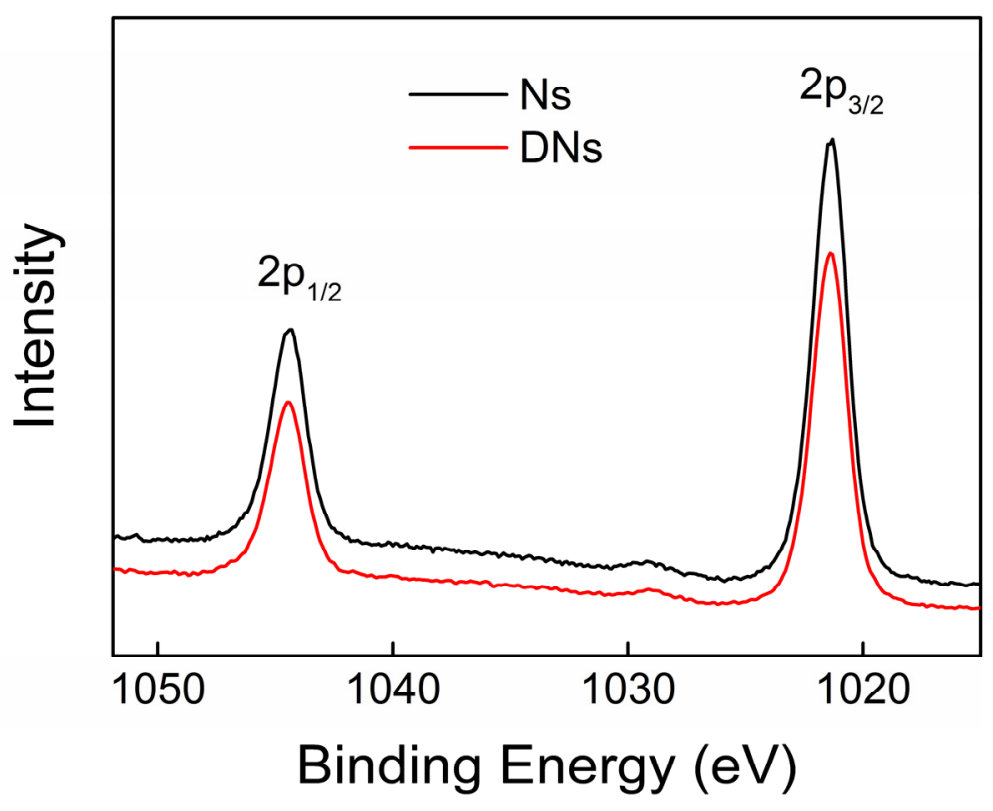

Figure S2. XPS spectrum of $\mathrm{Zn} 2 \mathrm{p} 1 / 2$ and $2 \mathrm{p} 3 / 2$ for ZIF-8 NPs (Ns) and DOX-encapsulated ZIF-8 NPs (DNs). 


\section{The size analysis of CDHNs in different solution}

The stability of the nanoparticles was also investigated by measuring the size distribution of CDHNs dispersed in $\mathrm{H}_{2} \mathrm{O}$, PBS or DMEM for different periods. As shown in Figure S3, the size distribution of CDHNs slightly changed with the incubation of different solutions for even $72 \mathrm{~h}$, indicating the good stability and dispersion of the nanoparticles.

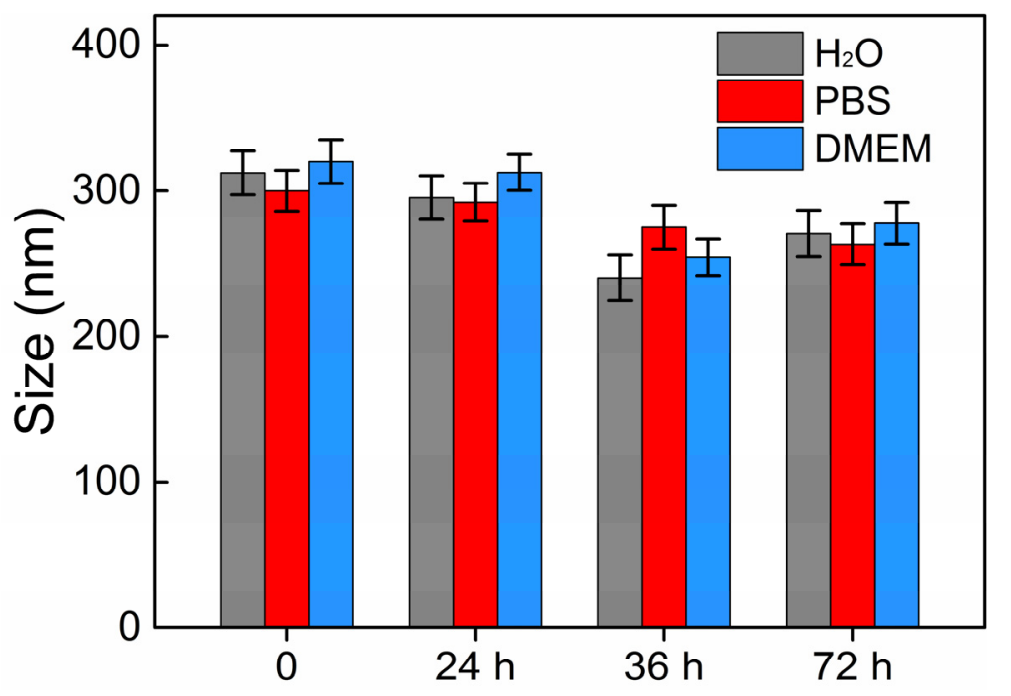

Figure S3. The size analysis of CDHNs in $\mathrm{H}_{2} \mathrm{O}$, PBS or DMEM for different incubation time by dynamic light scattering. Results are presented as means \pm standard deviation (SD) $(n=3)$. 


\section{UV-vis absorbance spectra of $\mathrm{Ce} 6$ and DOX in the presence of zinc ions}

The absorption spectra of $\mathrm{Ce} 6$ and DOX in the presence of zinc ions were provided and presented in Figure S4. The characteristic spectra of Ce6 shifted from $404 \mathrm{~nm}$ to $411 \mathrm{~nm}$ after the introduction of zinc ions (Figure S4A), attributed to the coordination interaction between $\mathrm{Ce} 6$ and $\mathrm{Zn}^{2+}$. However, the characteristic spectra of DOX remained unchanged with zinc ions (Figure S4B). The formation of the coordination bond between DOX and $\mathrm{Zn}^{2+}$ could be demonstrated by the change of binding energy peaks of $\mathrm{Zn} 2 \mathrm{p}$ (Figure S2). Meanwhile, aggregation of Ce6 and DOX in the nanoparticles may also contribute to the shift.
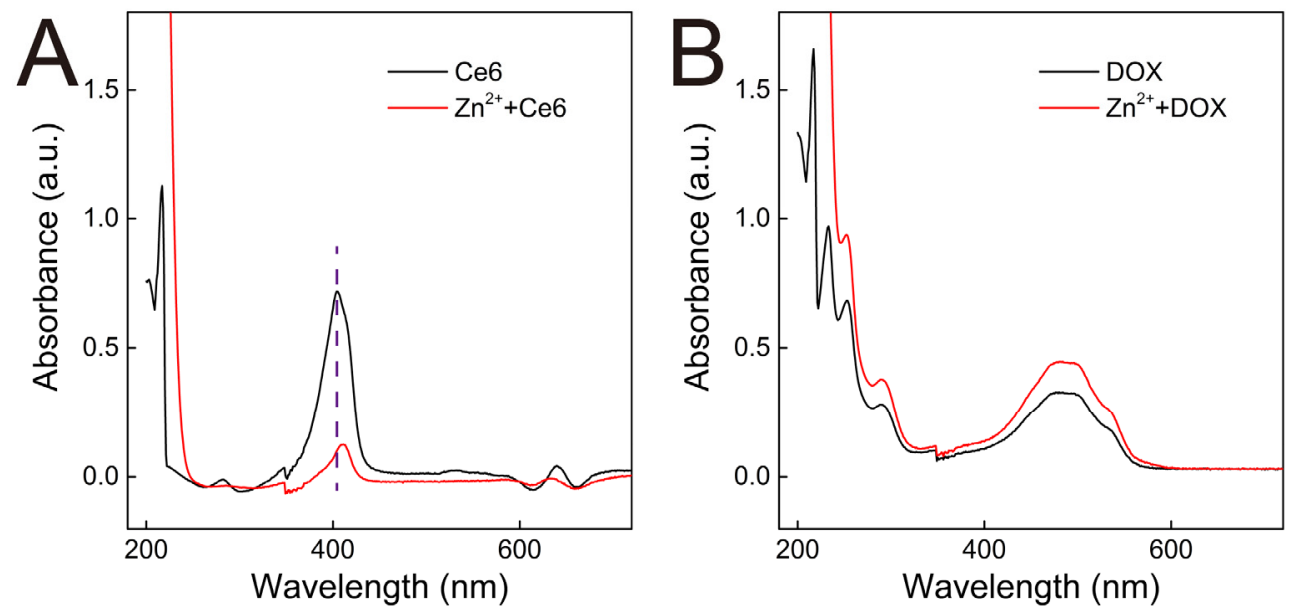

Figure S4. UV-vis absorbance spectra of Ce6 (A) and DOX (B) in the presence of zinc ions. 
The characteristic UV-vis absorption peak of siRNA

The characteristic UV-vis absorption peak of siRNA was shown in CDHNs at 260 $\mathrm{nm}$, while it was not observed in CDNs (Figure S5), indicating that siRNA was effectively incorporated in ZIF-8 nanoparticles.

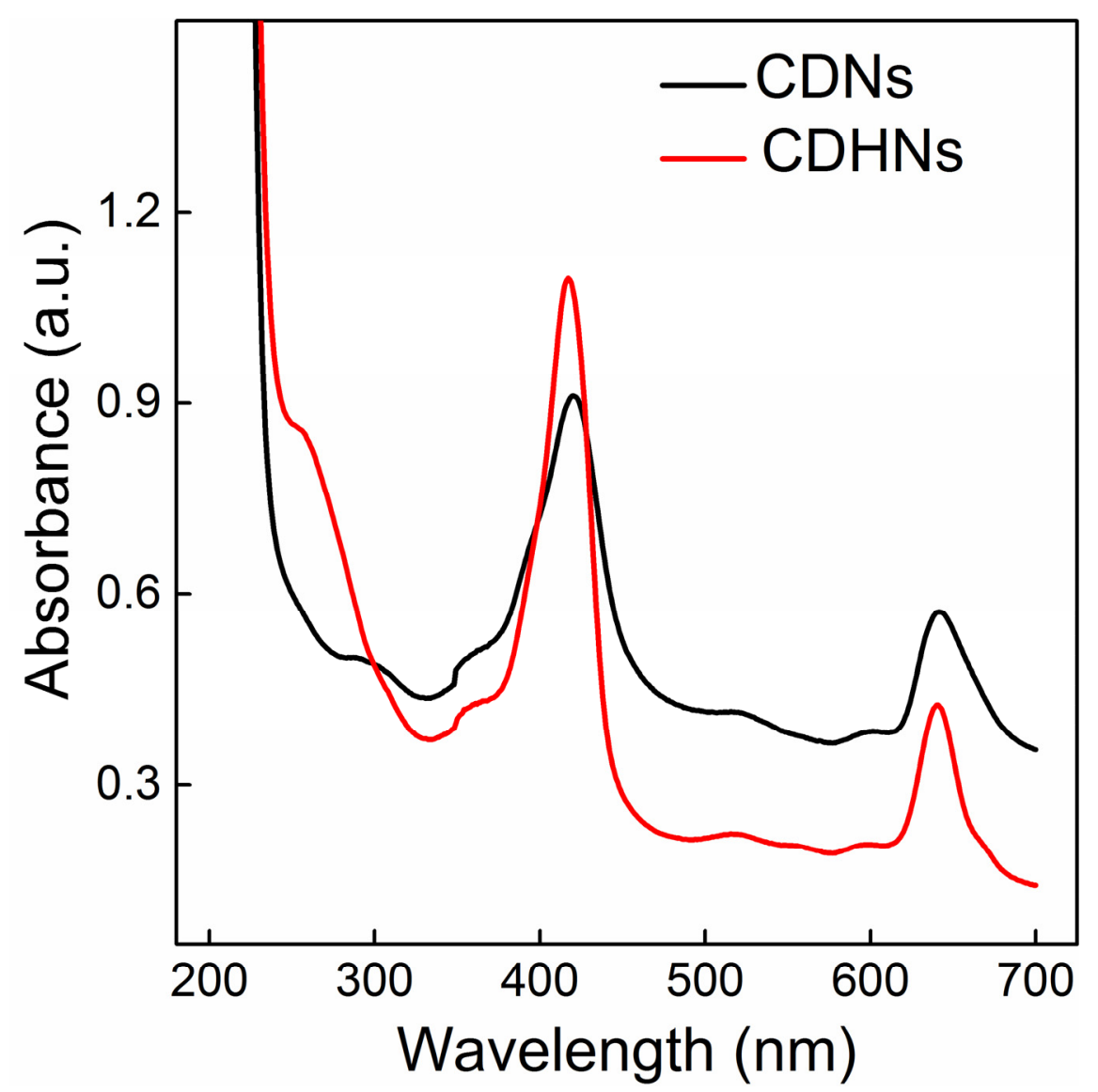

Figure S5. UV-vis absorption spectra of CDNs and CDHNs. 


\section{Supporting Information}

\section{The drug loading capacity and efficiency of different payloads}

To determine the encapsulated payloads in ZIF-8 nanoparticles, the calibration curve of the absorption intensity of DOX and Ce6 and fluorescence intensity of FAM-labeled siRNA (FAM-siRNA) were obtained with fitting equations (Figure S6). The drug loading capacity of Ce6, DOX, and FAM-siRNA was calculated to be $12 \mathrm{wt} \%$, 7 wt $\%$, and 3 wt $\%$, respectively. The drug loading efficiency of Ce6, DOX, and FAM-siRNA were calculated to be $64 \%, 38 \%$, and $95 \%$, respectively. The detailed calculation process is as follows:

1. The absorbance intensity of the released Ce6 was 0.52 a.u., and the corresponding concentration is calculated to be $14.0 \mu \mathrm{M}$ through the fitting equation (Figure S6A). Since the concentration of the Ce6 solution was diluted 100-fold before measurement, the real concentration of the encapsulated Ce6 should be $1.4 \mathrm{mM}$.

Drug loading capacity $($ DLC $)=$ mce6 $/ \mathrm{mcDHNs}^{2}=1.4 \mathrm{mM}^{*} 596.7 \mathrm{~g} \mathrm{~mol}^{-1} / 7.1 \mathrm{~g}$ $L^{-1}=12$ wt $\%$

Drug loading efficiency $(\mathrm{DLE})=\mathrm{C}_{\mathrm{Ce} 6} / \mathrm{C}_{\text {total }} \mathrm{Ce} 6=1.4 \mathrm{mM} / 2.2 \mathrm{mM}=64 \%$

2. The absorbance intensity of the released DOX was 0.37 a.u., and the corresponding concentration is calculated to be $31 \mu \mathrm{M}$ through the fitting equation (Figure S6B). Since the concentration of the DOX solution was diluted 30-fold before measurement, the real concentration of the encapsulated DOX should be $0.9 \mathrm{mM}$.

Drug loading capacity $(\mathrm{DLC})=\operatorname{mDox} / \mathrm{mCDHNs}=0.9 \mathrm{mM}^{*} 543.52 \mathrm{~g} \mathrm{~mol}^{-1} /$ $7.1 \mathrm{~g} \mathrm{~L}^{-1}=7 \mathrm{wt} \%$

Drug loading efficiency $($ DLE $)=\mathrm{C}_{\text {DOx }} / \mathrm{C}_{\text {total DOX }}=0.9 \mathrm{mM} / 2.4 \mathrm{mM}=38 \%$

3. The fluorescence intensity of the released FAM-siRNA was 239.3 a.u., and the corresponding concentration is calculated to be $0.95 \mu \mathrm{M}$ through the fitting equation (Figure S6C). Since the concentration of the FAM-siRNA solution was diluted 16-fold before measurement, the real concentration of the encapsulated FAM-siRNA should be $15.2 \mu \mathrm{M}$.

Drug loading capacity $($ DLC $)=m_{F A M-s i R N A} / m_{C D H N s}=15.2 \mu M^{*} 12724.1 \mathrm{~g} \mathrm{~mol}^{-1}$ / $7.1 \mathrm{~g} \mathrm{~L}^{-1}=3 \mathrm{wt} \%$

Drug loading efficiency $($ DLE $)=$ CFAM-siRnA $_{\text {f C }}$ total FAM-siRnA $=15.2 \mu \mathrm{M} / 16 \mu \mathrm{M}$ $=95 \%$ 

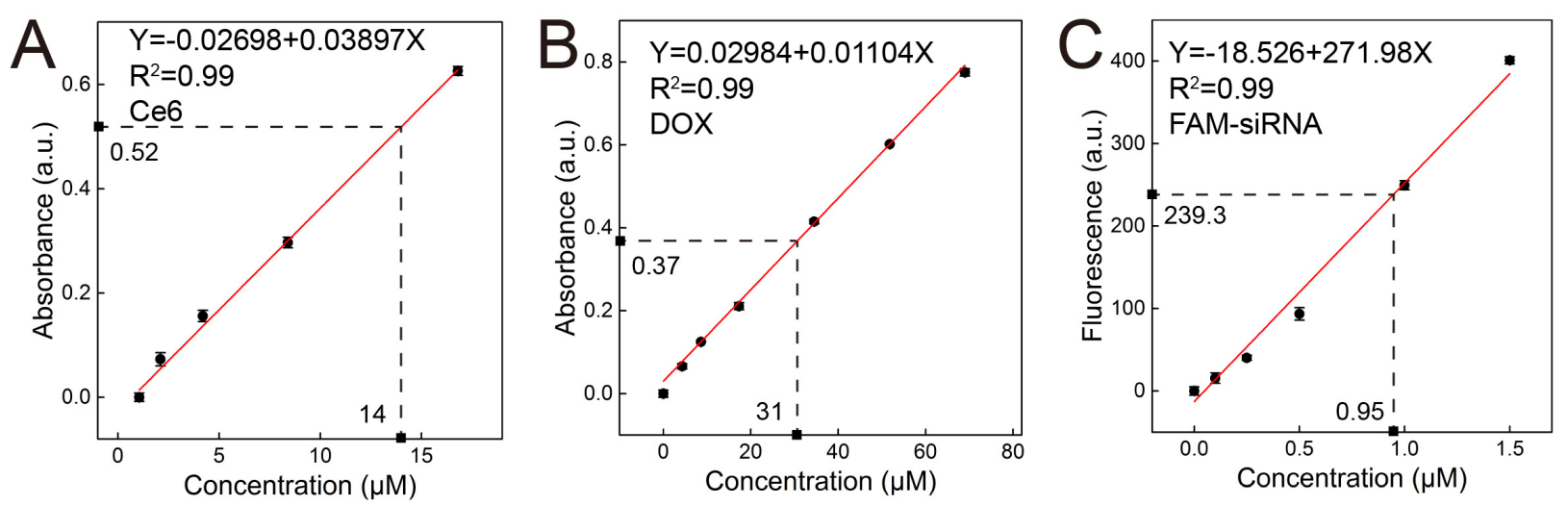

Figure S6. The calibration curve of the absorption intensity of DOX (A) and Ce6 (B) and fluorescence intensity of siRNA (C). 


\section{The effect of ROS on the integrity of siRNA in CDHNs}

To investigate whether the implementation of PDT would affect the integrity of the encapsulated siRNA in CDHNs, we analyze the PAGE brand of siRNA in the acidic dissociated CDHNs with different laser irradiation (Figure S7). With the increase of laser power density, no damaged siRNA fragment was observed in any brand, indicating the generated ROS had no effect on the integrity of siRNA.

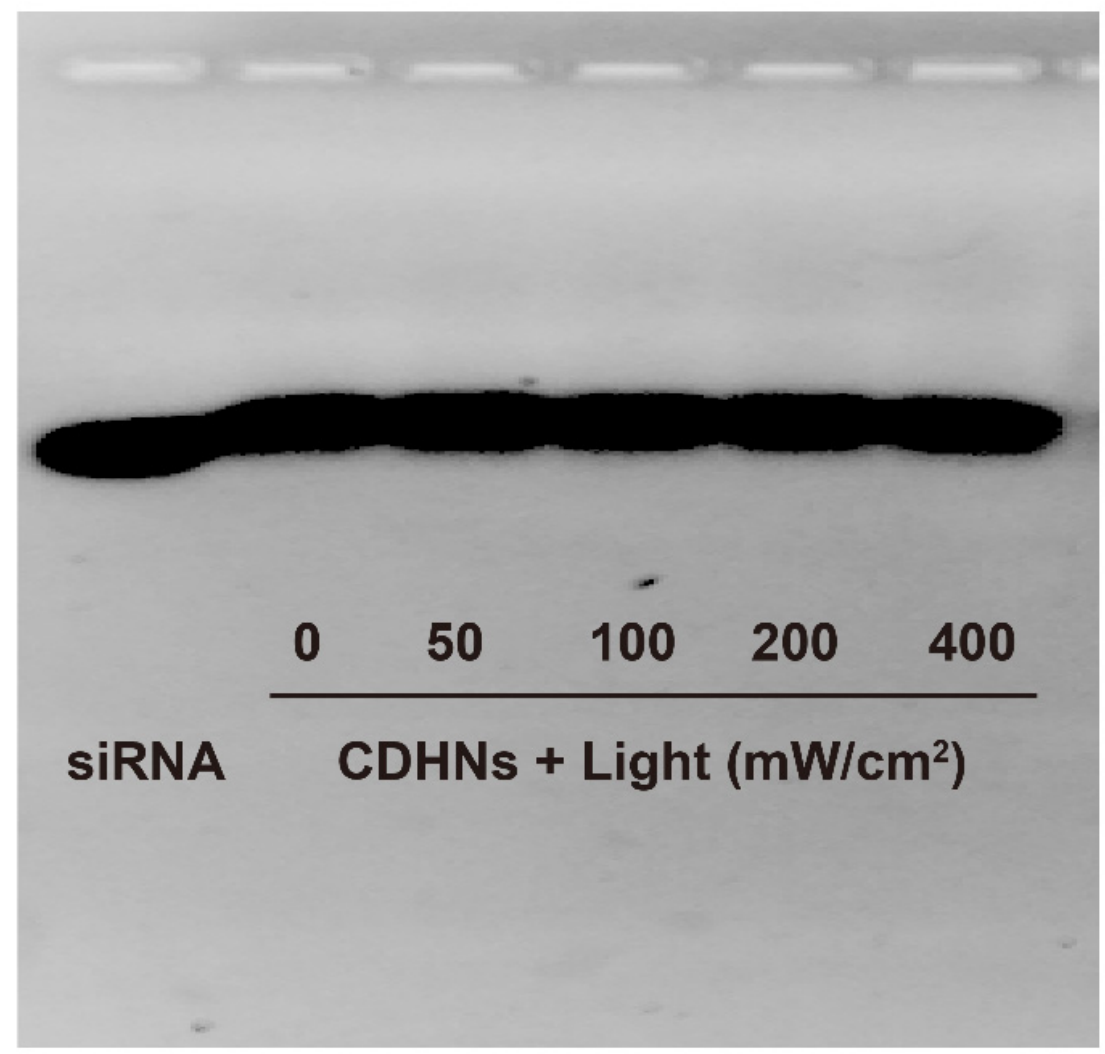

Figure S7. The PAGE analysis of CDHNs in acidic buffer under different laser irradiation density. 


\section{Cytotoxicity of CNs on MCF-10A and MDR/MCF-7 cells}

The cytotoxicity of CNs on MCF-10A and MDR/MCF-7 cells was investigated by standard 3-(4,5-cimethylthiazol-2-yl)-2,5-diphenyl tetrazolium bromide (MTT) assay. As shown in Figure S8, the cell viability remained $93 \%$ and $96 \%$ even with $40 \mu \mathrm{g} / \mathrm{mL}$ nanoparticles for $72 \mathrm{~h}$ in MCF-10A and MDR/MCF-7 cells, respectively, indicating the good biocompatibility of CNs. The optimized concentration of CNs nanoparticles was $40 \mu \mathrm{g} / \mathrm{mL}$ and it was used for the following intracellular experiments.

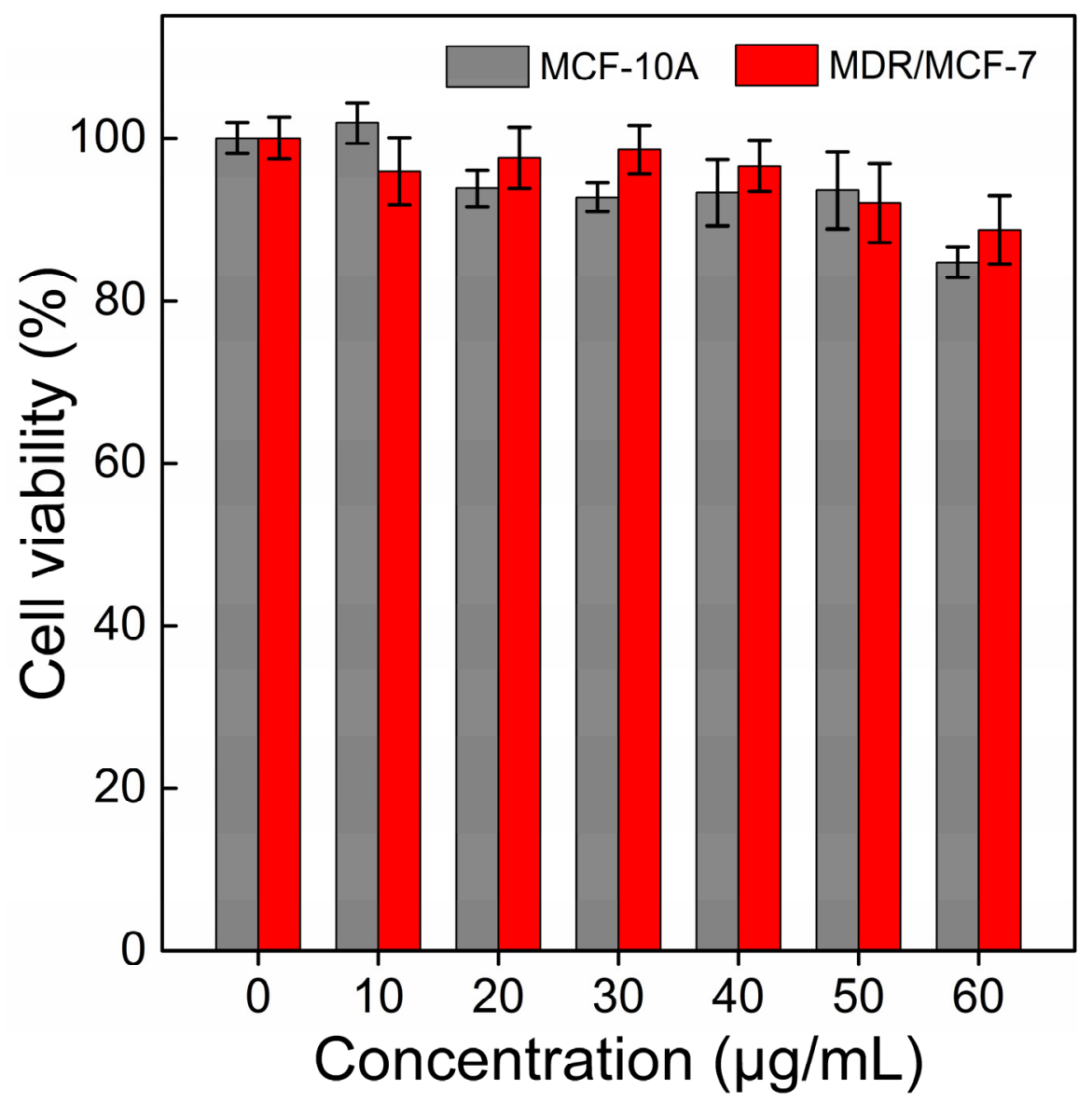

Figure S8. Cytotoxicity of CNs on MCF-10A and MDR/MCF-7 cells with different concentrations of $\mathrm{CNs}$ for $72 \mathrm{~h}$. 


\section{Optimization of incubation time}

The cellular uptake behavior of CNs was investigated by flow cytometry. With the increasing incubation time, the fluorescence of $\mathrm{CNs}$ reached a plateau after $2 \mathrm{~h}$ (Figure S9), which was chosen as the optimized incubation time for the following in vitro experiments.

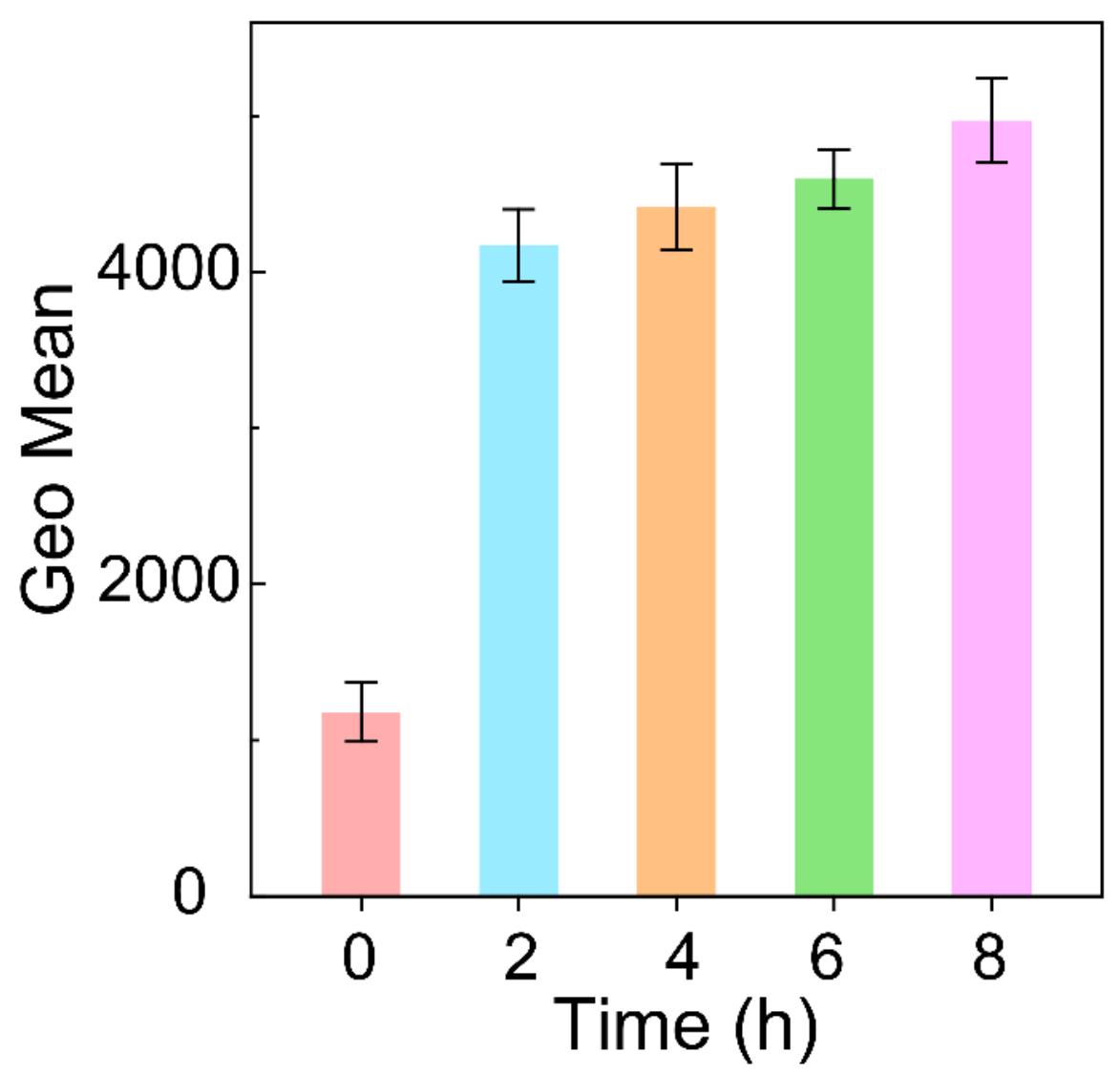

Figure S9. Statistical analysis of the intracellular fluorescence intensity of CNs-treated MDR/MCF-7 cells for $0,2,4,6$, and $8 \mathrm{~h}$ by flow cytometry. 


\section{Intracellular uptake of CNs by CLSM}

CLSM was utilized for investigating the intracellular uptake of free $\mathrm{Ce} 6$ and CNs. The intracellular green fluorescence was only observed in CNs-treated MDR/MCF-7 cells but not free Ce6-treated cells, and it was surrounded by the red membrane dye (Figure S10A). The cellular uptake behavior of CNs in different organelles was investigated by CLSM with different organelles-specific dyes, such as HOECHST for nucleus, and Lyso Tracker Red for lysosome in Figure S10B. The magnification of the CLSM image showed that most of the CNs were dispersed in lysosomes with the colocation coefficient of 0.602 , indicating that CNs were internalized by endocytosis. These results demonstrated the efficient intracellular delivery of Ce6 by ZIF-8 nanocarriers and CNs were internalized by endocytosis.

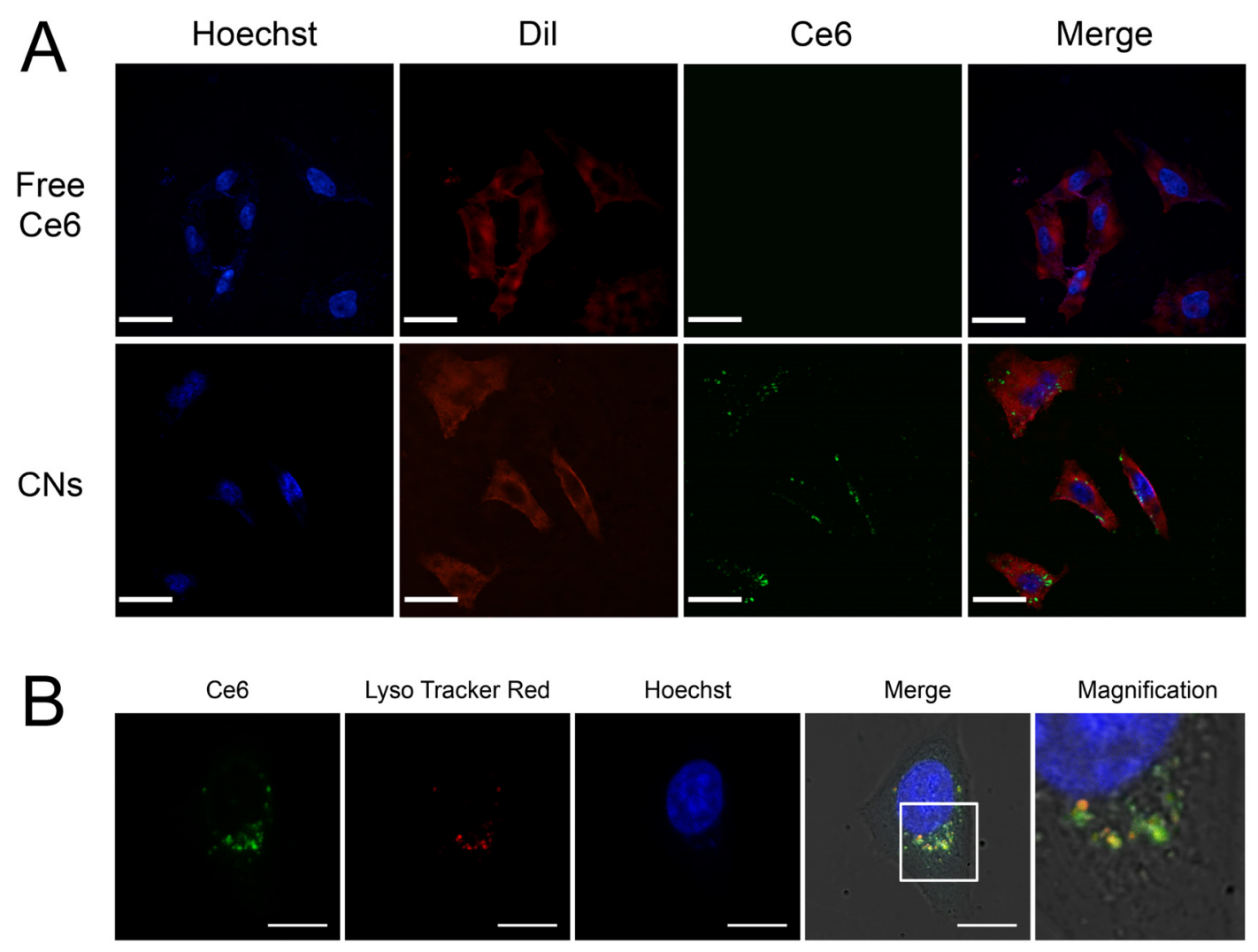

Figure S10. (A) Confocal fluorescence images of MDR/MCF-7 cells that were incubated with free Ce6 and CNs $(40 \mu \mathrm{g} / \mathrm{mL})$. Nuclei and membrane were stained with Hoechst and Dil, respectively. Scale bar $=20 \mu \mathrm{m}$. (B) The endocytic uptake of CNs via CLSM. Nuclei and lysosomes were stained with Hoechst and Lyso Tracker Red, respectively. Scale bar $=10 \mu \mathrm{m}$. 


\section{The accelerated lysosomal escape of $\mathrm{Ce} 6$ under irradiation}

The subcellular co-localization of Ce6 with lysosomes (stained with lyso-tracker red) significantly decreased under photoirradiation (Figure S11), indicating that PDT could effectively promote the lysosomal escape of therapeutics.

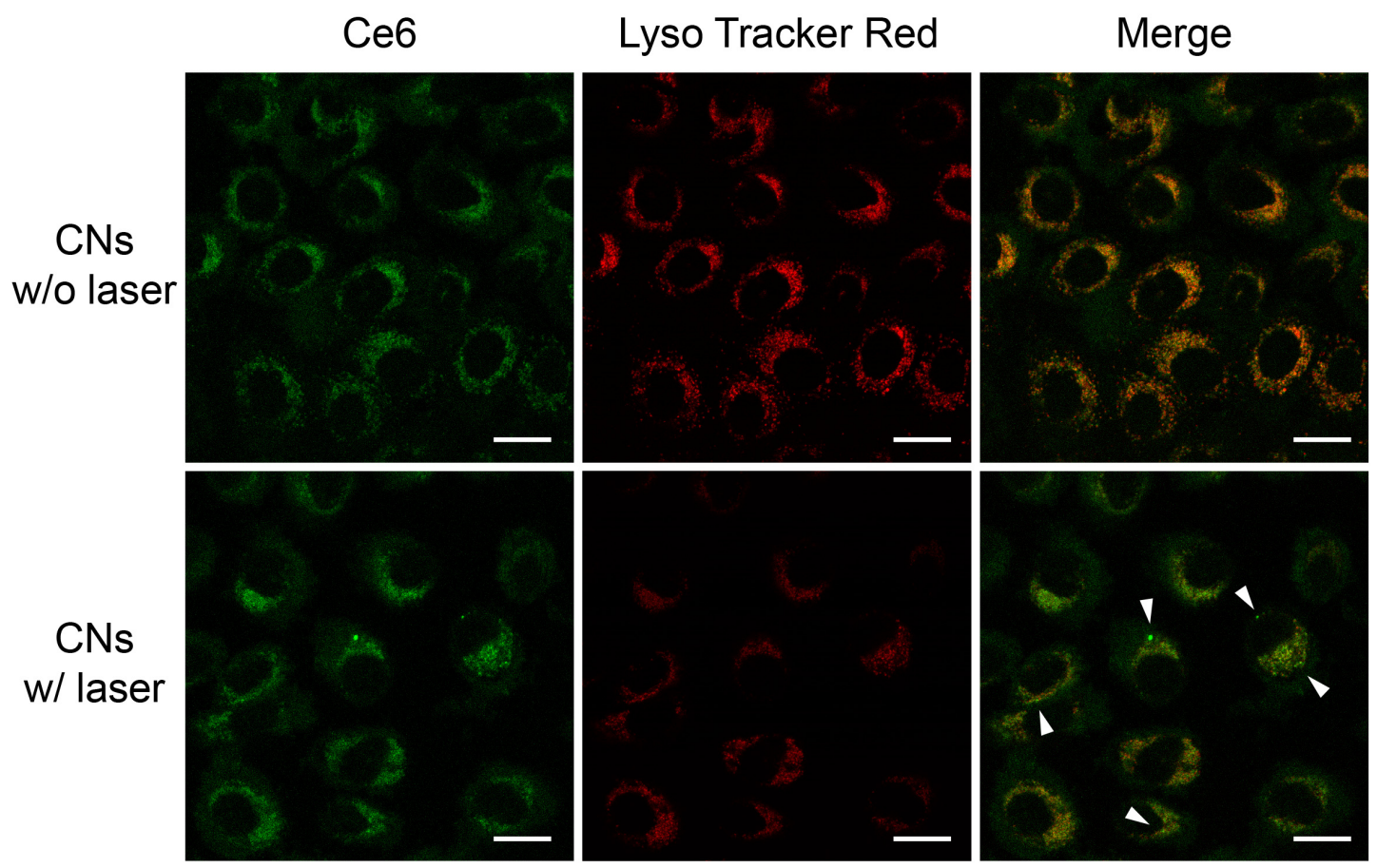

Figure S11. CLSM imaging of CNs-treated MDR/MCF-7 cells with or without photoirradiation. Nuclei and lysosomes were stained with Hoechst and lyso-tracker red, respectively. Scale bar $=20 \mu \mathrm{m}$. 


\section{The accelerated release of DOX by PDT}

To investigate whether the PDT-generated ROS could accelerate the concomitant DOX release, CLSM was utilized for investigating the intracellular ROS and DOX with different incubation time with or without photoirradiation. The intracellular green fluorescence of DCF and red fluorescence of DOX elevated with the increasing incubation time with photoirradiation. In addition, the red fluorescence was slightly enhanced after the photoirradiation treatment compared with that of the non-irradiation group under different incubation time (Figure S12). These results demonstrated the efficient accelerated release of DOX by the PDT-generated ROS.

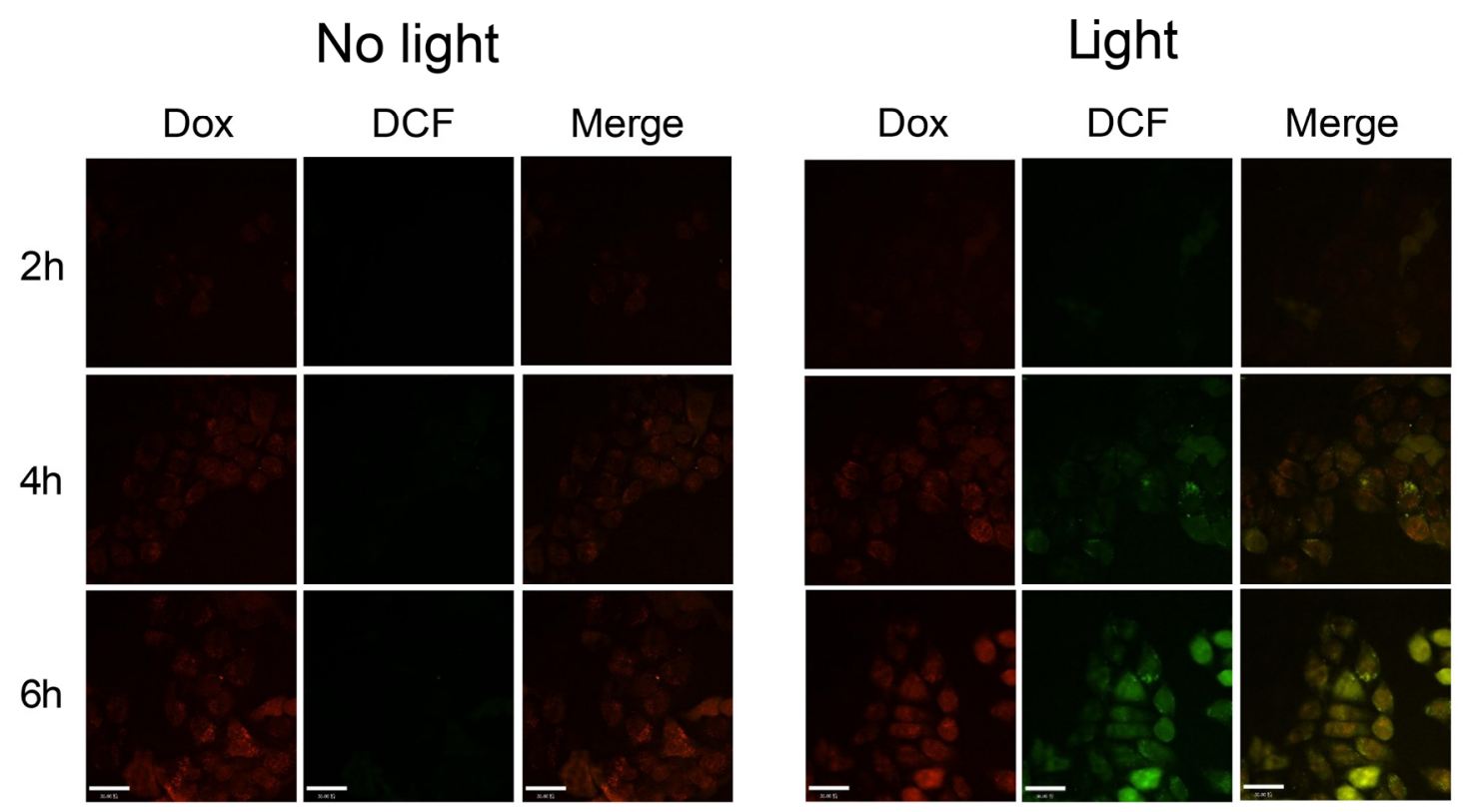

Figure S12. Confocal fluorescence images of MDR/MCF-7 cells that were incubated with CDHNs $(40 \mu \mathrm{g} / \mathrm{mL})$ without or with photoirradiation under different incubation time. Scale bar $=20 \mu \mathrm{m}$. 


\section{Scheme of our double-gained gene therapy (GT)}

Compared with the combined PARP siRNA-mediated PDT and P-gp siRNA-mediated PDT (where the PARP siRNA and P-gp siRNA could respectively eliminate the PDT- and CT-associated therapeutic resistance), our single HIF-1 $\alpha$ siRNA administration with double therapeutic gain strategy could be utilized to simultaneously relief chemophototherapy resistance (Figure S13) and has the following obvious advantages: (1) a more simplified all-in-one design without complicated and multistep preparation and optimization; (2) a higher efficient therapeutic performance for three reasons: (I) more significant and comprehensive synergistic therapeutic effect as revealed by the low CI value, rather than the combinational effect; (II) higher loading capacity of siRNA agent than that of two different siRNA agents since the total siRNA loading capacity is constant; (III) the reduced utilization of intracellular RNA-induced silencing complex (RISC)-related proteins, including Argonaute and Dicer enzymes; (3) a remarkable improved anti-interference performance since the encapsulation of two different siRNAs would increase their vulnerability to the external disturbance and the possible inter-cross-talk interference.

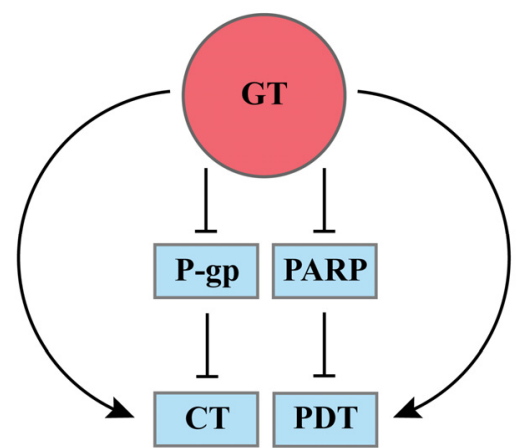

Figure S13. Scheme of our double-gained gene therapy (GT) for overcoming photochemotherapy resistance simultaneously. 


\section{The annexin V-FITC/PI-staining method}

The flow cytometry analysis of MDR/MCF-7 cells with different treatments was investigated by annexin V-FITC and PI staining in Figure S14. With the combination of siRNA-mediated GT, the late cell apoptosis/necrosis of PDT $(3.14 \%, 7.01 \%)$ and CT $(3.44 \%, 7.86 \%)$ could be promoted to GT+PDT $(4.07 \%, 12.0 \%)$ and GT $+\mathrm{CT}(3.68 \%$, $14.8 \%$ ), respectively. Obviously, the additional HIF-1 $\alpha$ siRNA could significantly improve the therapeutic effect of PDT and CT. And the triple combined GPC (photoirradiated CDHNs) realized the most efficient cell late apoptosis/necrosis $(4.78 \%, 25.8 \%)$, demonstrating the most effective pro-apoptotic effect of CDHNs on cancer cells. These results demonstrated that the effective therapeutic CDHNs nanoplatform could be used in multimodal cancer therapy through the mutual enhancement of PDT, CT, and GT, where GT might play a more important role in this process.
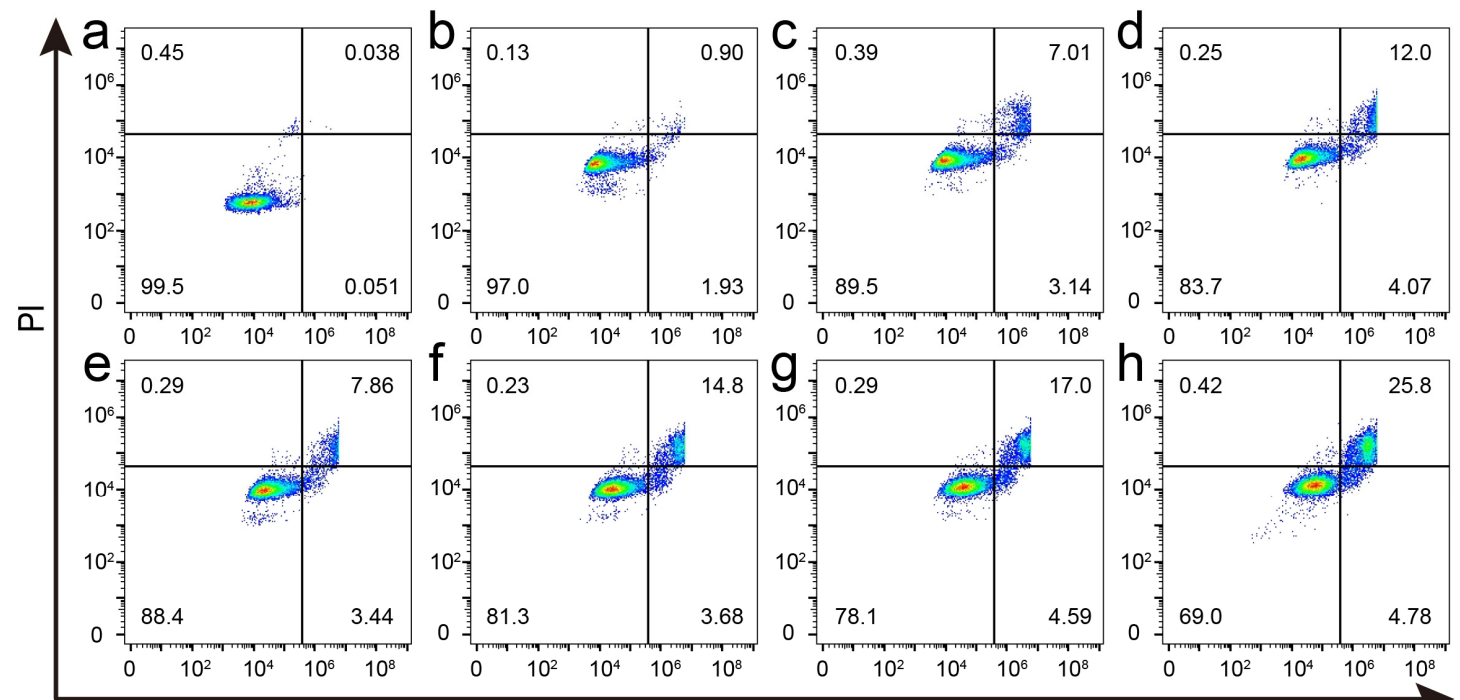

Annexin V

Figure S14. Flow cytometry analysis of MDR/MCF-7 cell apoptosis with different treatments: PBS (a), free DOX (b), photoirradiated CNs (c), photoirradiated CHNs (d), CDNs (e), CDHNs (f), photoirradiated CDNs (g), and photoirradiated CDHNs (h). 


\section{Cell cycle distribution assay}

Cell cycle assay was carried out to investigate the mechanism of the therapeutic effect (Figure S15). Treating MDR/MCF-7 cells with $\mathrm{CNs}+$ Light (C) or CDNs (E) resulted in an increased S population and decreased G2/M population compared with the control group (A). In addition, more cell arrested at the S phase after the GT-PDT (D) or GT-CT (F) treatment. Specifically, the G2/M population decreased nearly 1-fold upon entry into the $\mathrm{S}$ phase for the cells treated with $\mathrm{CDHNs}+$ Light $(\mathrm{H})$ compared to the control group and thus greatly induced cell cycle arrest. The cell cycle redistribution implied that synergistic therapy could inhibit cell proliferation significantly.
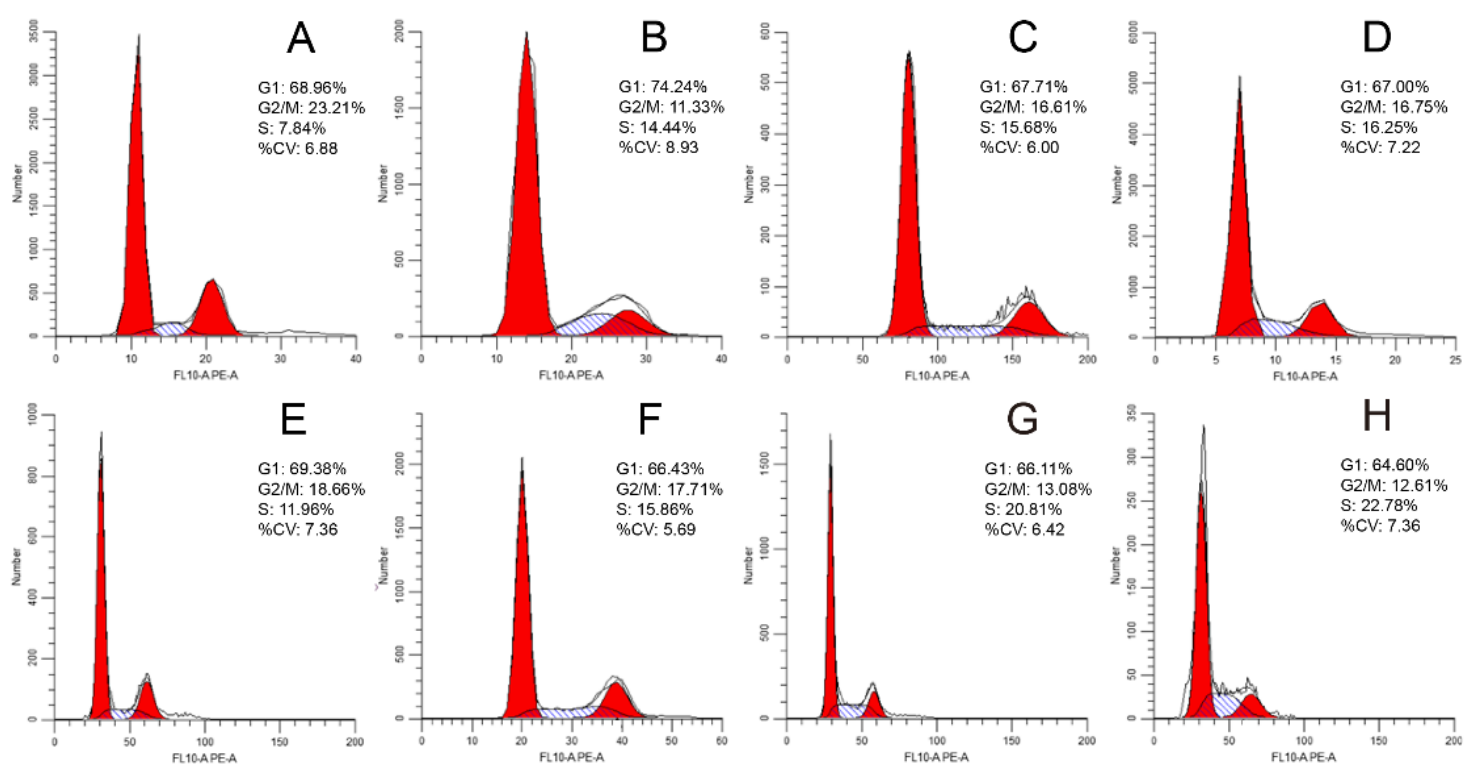

Figure S15. Cell cycle distribution of MDR/MCF-7 cells with different treatments: PBS (A), free DOX (B), CNs + Light (C), CHNs + Light (D), CDNs (E), CDHNs (F), CDNs + Light $(\mathrm{G})$, and CDHNs + Light $(\mathrm{H})$. 


\section{Synergism between PDT and CT}

The synergism between PDT and CT was investigated by CI values of photoirradiated CDNs (CP) at different concentrations (Figure S16). About 0.5 of CI value was calculated between PDT and CT, demonstrating the slight synergism between PDT and CT. This might be attributed to the promotion of the lysosome escape for boosting drug release by PDT-generated reactive oxygen species (ROS).

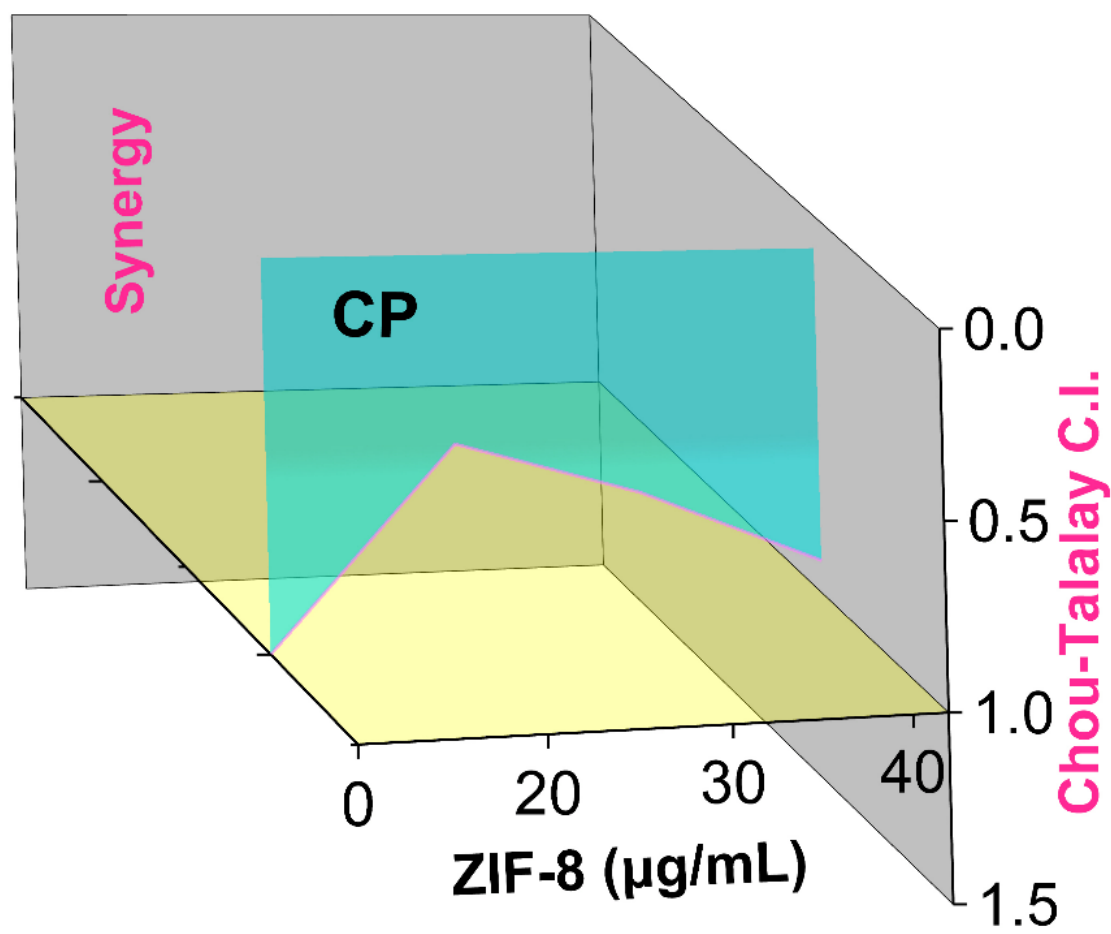

Figure S16. CI values of photoirradiated CDNs (CP) at different concentrations. 


\section{Hemolysis test of CDHNs}

Hemolysis test was carried out to investigate the biocompatible of the CDHNs. No hemolytic reaction was observed with even as high as $200 \mu \mathrm{g} / \mathrm{mL}$ of CDHNs (Figure S17), indicating the good compatibility of the nanocarriers for intravenous injection.

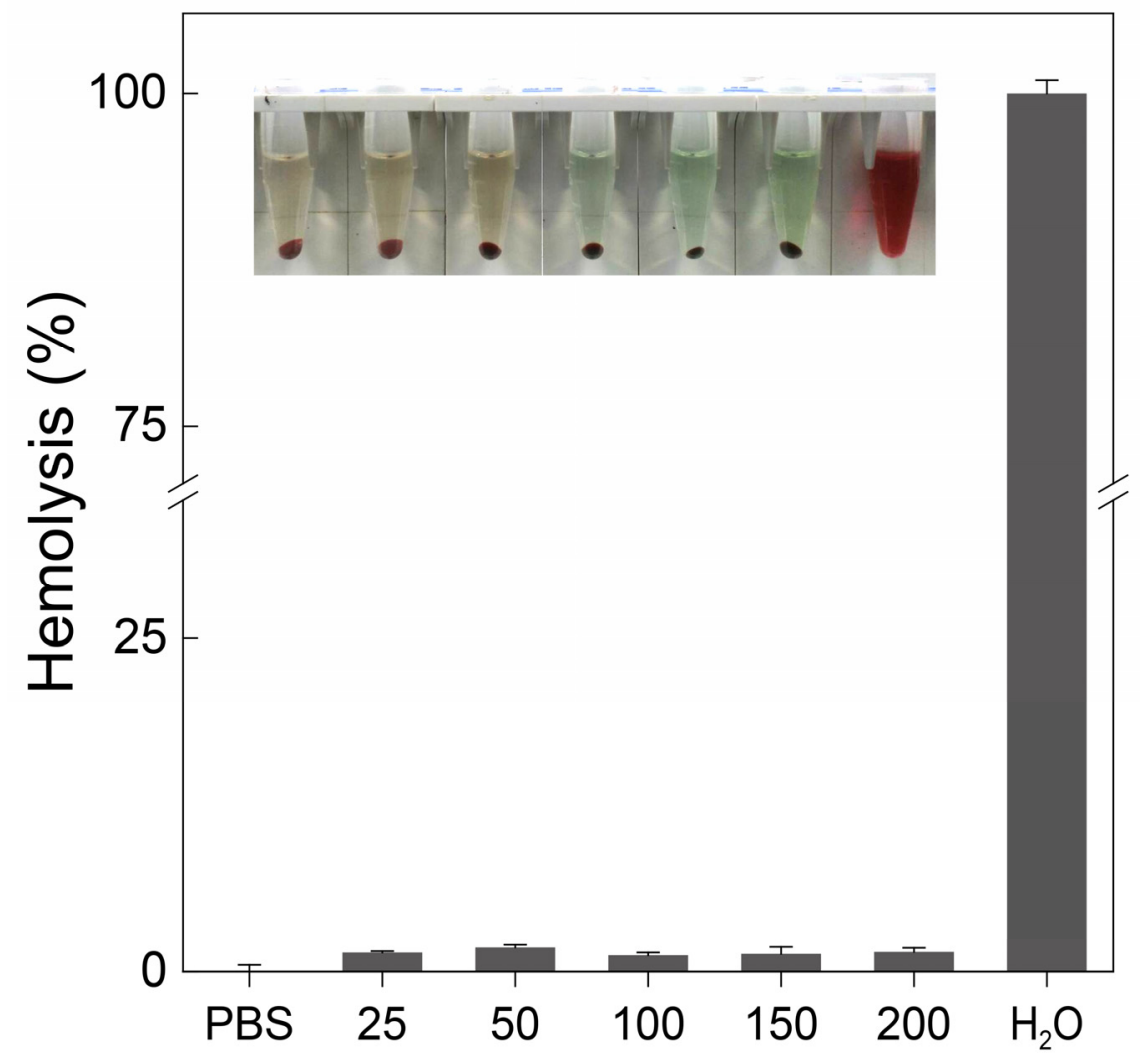

Figure S17. Hemolysis test of CDHNs at different concentrations. 


\section{Supporting Information}

\section{H\&E staining of CDHNs in major organs}

To investigate the potential toxicity of CDHNs on major organs, the differently treated mice were sacrificed and the collected heart, liver, spleen, lung, and kidney were analyzed by using hematoxylin and eosin (H\&E) staining. No physiological abnormality was observed in most of the major organs with different treatments. However, besides $(\mathrm{CHNs}+$ Light)-, CDHNs- and (CDHNs + Light)-treated livers, livers in other groups showed small nodules (Figure S18), demonstrating the minimal systematic toxicity of the HIF-1 $\alpha$ siRNA-containing nanoparticles.

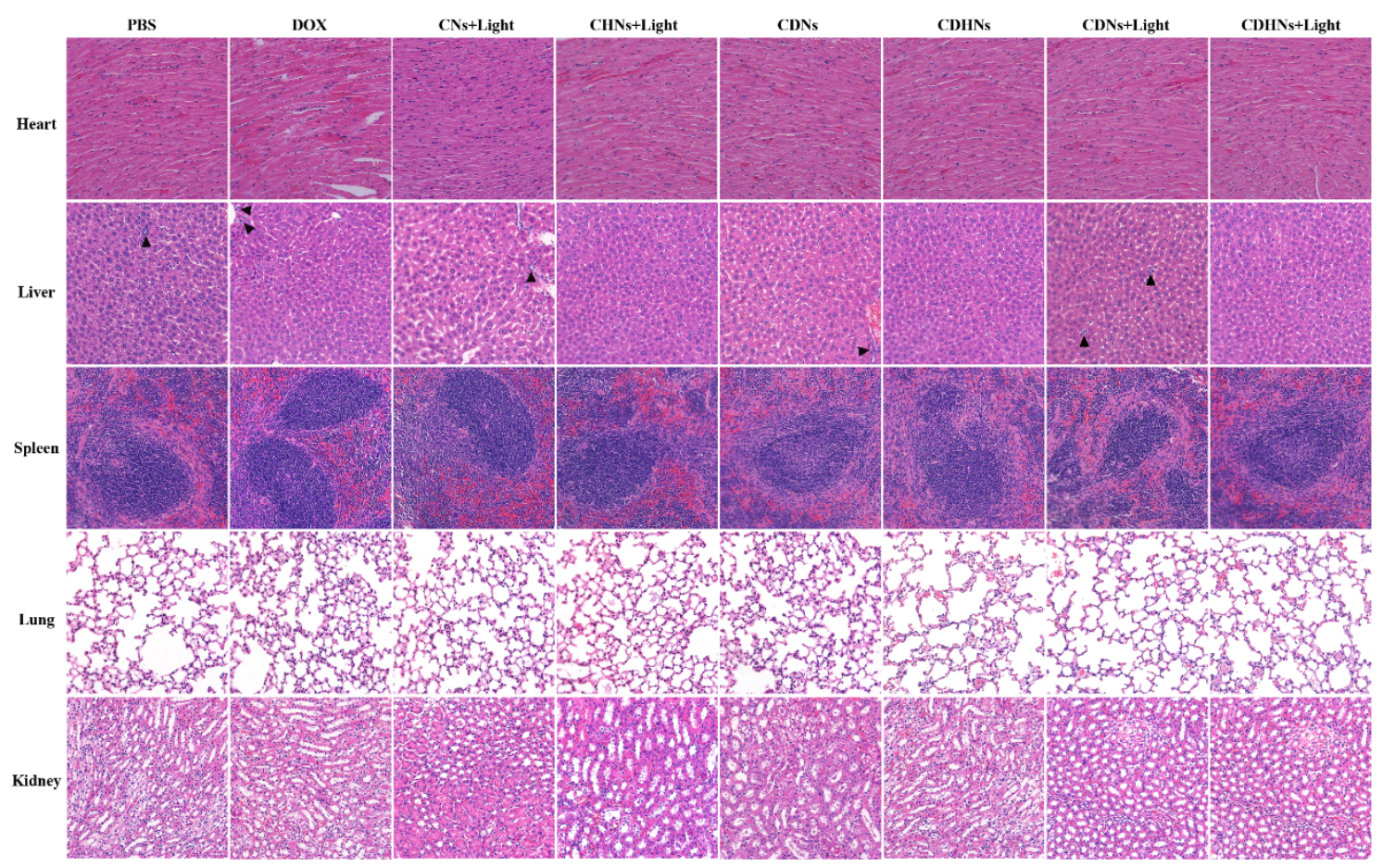

Figure S18. Representative H\&E staining of main organs of differently treated mice, including heart, liver, spleen, lung, and kidney, which were collected from MDR/MCF-7 tumor-bearing female nude mice after intravenous administration of different materials $(10 \mathrm{mg} / \mathrm{kg})$. Detailed information is provided in the experimental section. 


\section{Serum biochemistry analysis of CDHNs}

Serum biochemistry analysis was also used to explore the potential systematic toxicity of CDHNs. All of these key parameters of the nanoparticles-treated mice were in good accordance with that of healthy mice (Figure S19), indicating minimal systematic toxicity of the proposed nanoparticles.
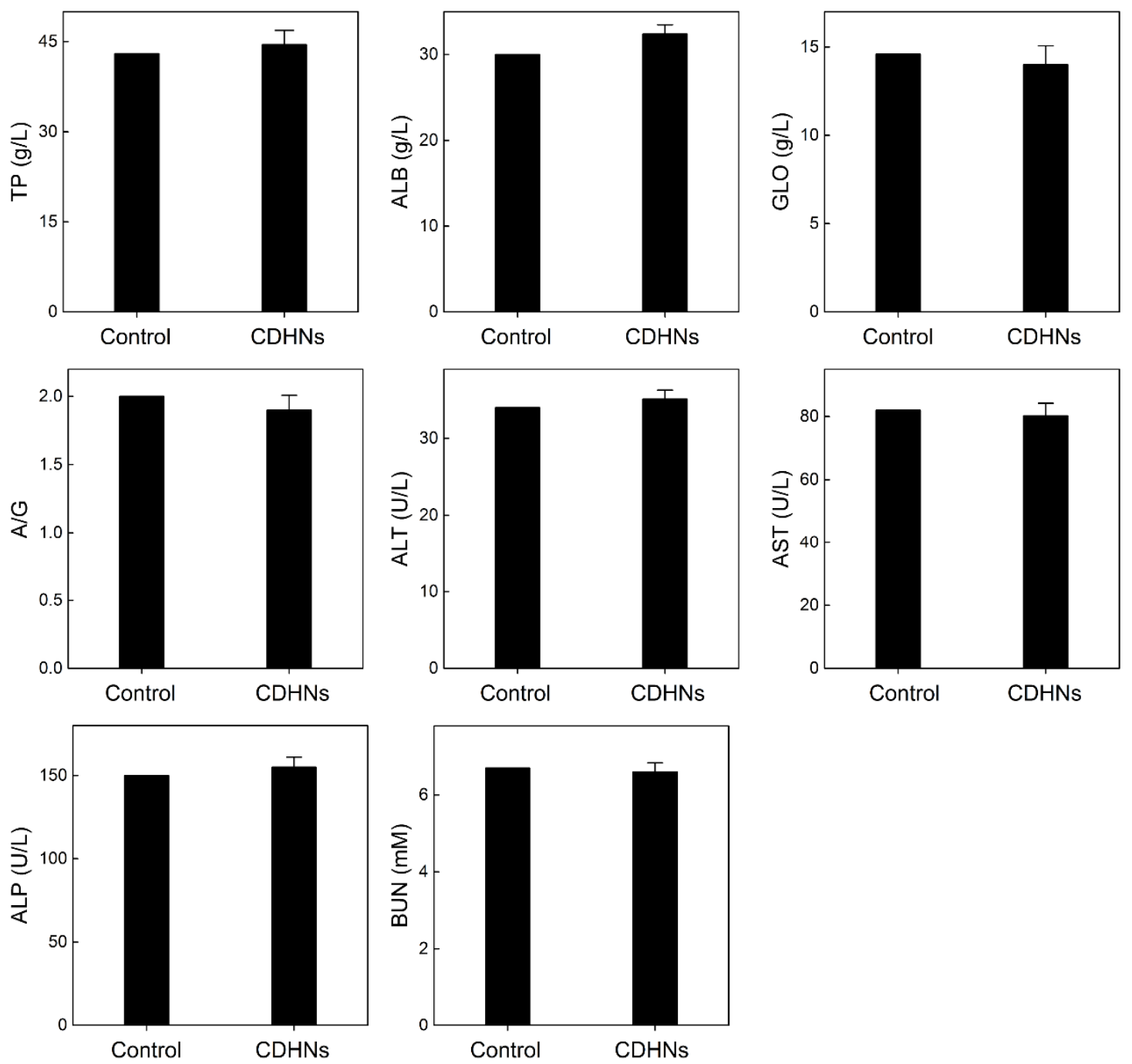

Figure S19. Blood biochemistry analysis of mice that underwent with and without CDHNs treatment $(10 \mathrm{mg} / \mathrm{kg})$ for $24 \mathrm{~h}$. The blood biochemical levels include total protein (TP), albumin (ALB), globulin (GLO), albumin and globulin ratio (A/G), alanine aminotransferase (ALT), aspartate aminotransferase (AST), alkaline phosphatase (ALP) and urea nitrogen (BUN). Detailed information is provided in the experimental section. 


\section{In vivo imaging analysis of CDHNs}

In vivo imaging system was carried out to investigate the biodistribution of CDHNs. The CDHNs-treated mice showed accumulated fluorescence of Ce6 in tumor site (Figure S20), demonstrating the tumor-specific accumulation of CDHNs in MCF-7 tumor-bearing mice.

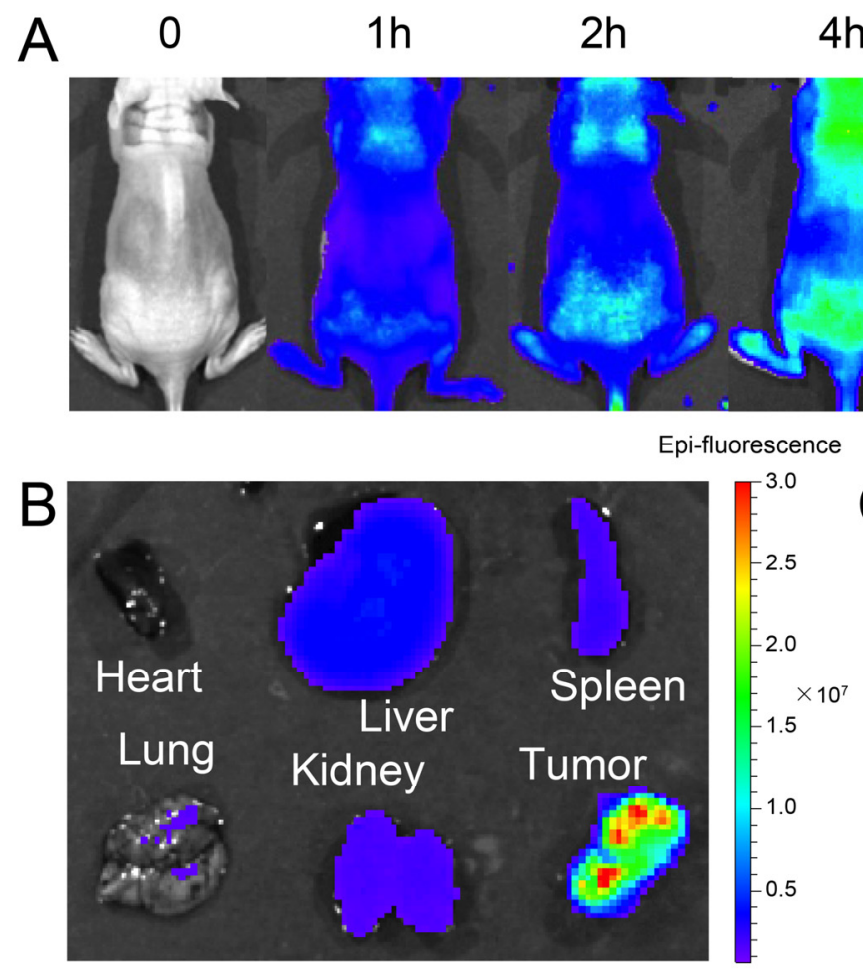

Radiant Efficiency

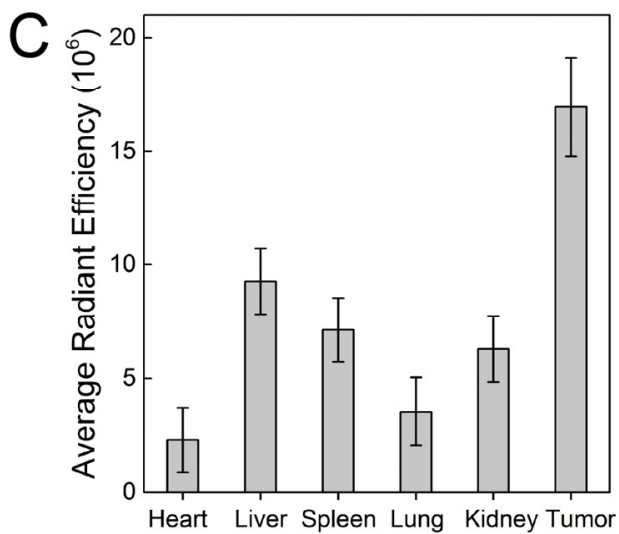

Figure S20. (A) In vivo fluorescence imaging of mice bearing MDR/MCF-7 tumor after intravenous injection of CDHNs $(10 \mathrm{mg} / \mathrm{kg}$ ) for different durations. (B) Ex vivo fluorescence imaging of major organs and tumors dissected from mice that were intravenously injected with CDHNs for 24 h. (C) Statistical analysis of the percentage of ex vivo fluorescence imaging of major organs and tumors. Results are presented as means \pm standard deviation $(\mathrm{SD})(\mathrm{n}=5)$. Detailed information is provided in the experimental section. 


\section{ICP-AES analysis of $\mathrm{Zn}$}

The in vivo long-term tissue biodistribution of CDHNs was investigated by inductively coupled plasma atomic emission spectroscopy (ICP-AES). As shown in Figure S21, $\mathrm{Zn}$ content in tumor significantly increased after $4 \mathrm{~h}$ post-injection of CDHNs, which was in accordance with the in vivo imaging experiment. It further demonstrated that the nanoparticles were suitable for the EPR effect. In addition, Zn was mainly accumulated in kidney and reticuloendothelial systems (RES) after $4 \mathrm{~h}$ post i.v. administration, while it obviously decreased with the extended injection time and stimulated in intestines, verifying that the CDHNs could be effectively eliminated without long-term physiological toxicity.

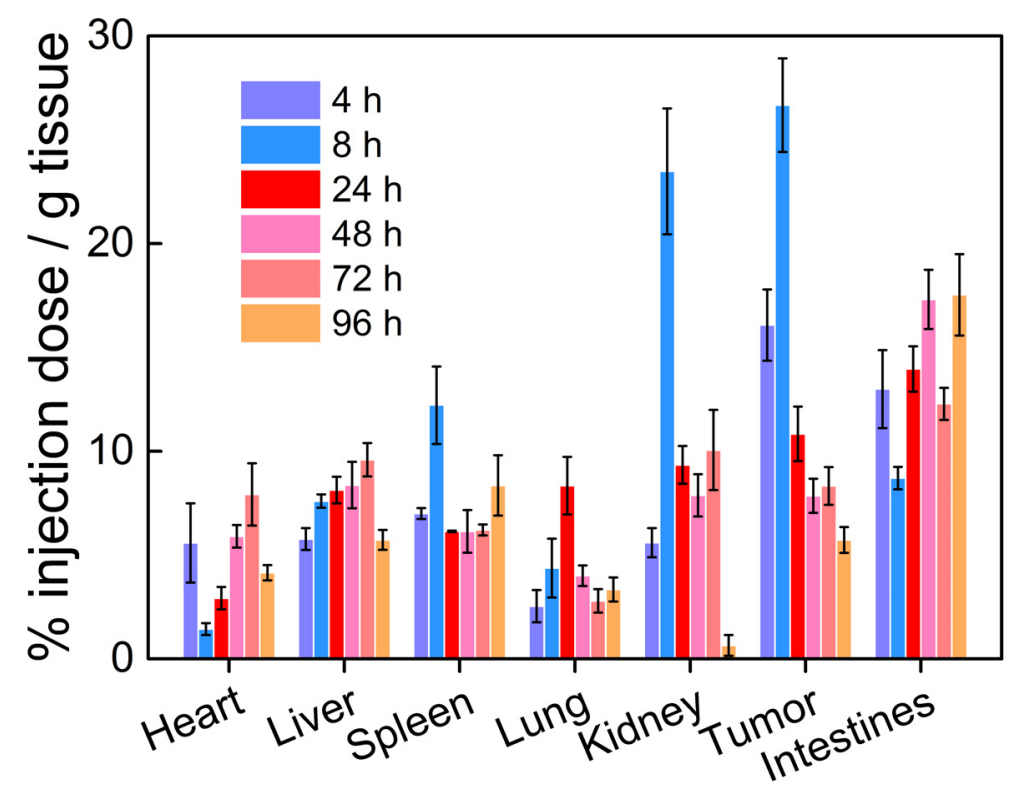

Figure S21. The in vivo long-term tissue biodistribution of CDHNs by inductively coupled plasmas atomic emissive spectrometry (ICP-AES) analysis of Zn. 18 MCF-7/ADR tumor-bearing BALB/c female nude mice were intravenous injected with Ns and then were euthanized at indicated time points $(4,8,24,48,72$ and $96 \mathrm{~h}, \mathrm{n}=3)$. 


\section{Immunofluorescence images of $\gamma-\mathrm{H}_{2} \mathrm{AX}$ and $\mathrm{HIF}-1 \alpha$ at the same site}

The immunofluorescence images of $\gamma-\mathrm{H} 2 \mathrm{AX}$ (red) and HIF-1 $\alpha$ (green) at the same site under different treatments were carried out to investigate the relationship between these two indicators. As shown in Figure S22, the more obvious red $\gamma-\mathrm{H}_{2} \mathrm{AX}$ immunofluorescence signal was shown in the photoirradiated CHNs group than that of the CNs group with photoirradiation and the same trend was observed in the CDHNs and CDNs groups, indicating the gene therapy could effectively suppress the reconstruction of PDT-involved dsDNA broken.
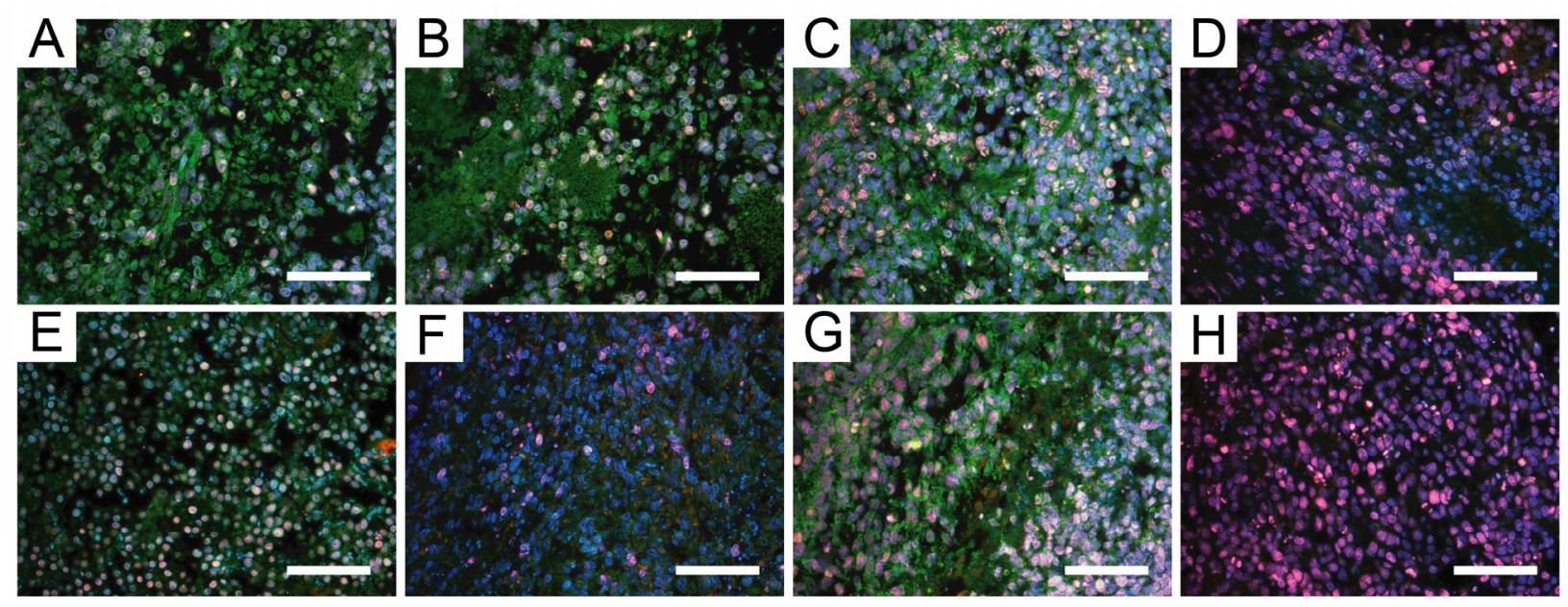

Figure S22. The immunohistochemical analysis of tumors after different treatments: PBS (a), free DOX (b), photoirradiated CNs (c), photoirradiated CHNs (d), CDNs (e), CDHNs (f), photoirradiated CDNs (g), and photoirradiated CDHNs (h). Scale bar $=100$ $\mu \mathrm{m}$. 